\title{
Inclusión Social y Laboral de la Mujer Gitana. El Aprendizaje-Servicio como Apuesta por la Innovación Educativa
}

\section{Social and Labour Inclusion of Gypsy Woman. Service-Learning as a Commitment to Educational Innovation}

\author{
Ana Amaro Agudo, Andrés Soriano Díaz, Nazaret Martínez-Heredia *, Gracia González-Gijón \\ Universidad de Granada, España
}

\begin{abstract}
DESCRIPTORES:
Aprendizaje-servicio

Innovación

Mujer gitana

Estudiantes

Justicia social

RESUMEN:

El presente trabajo muestra las aportaciones fundamentales de un Proyecto de Innovación Docente financiado por la Universidad de Granada durante el curso académico 2019/2020. Su doble finalidad ha permitido trabajar la formación-inclusión social de la mujer gitana que reside en la Zona Norte de la ciudad de Granada y, por otro lado, desarrollar competencias profesionales en el alumnado que cursa los grados de educación social y pedagogía. Para ello se ha puesto en práctica un proceso de trabajo colaborativo en el que han participado mujeres gitanas junto a profesionales de la educación social y de la pedagogía, así como alumnado de esos grados. Para llevar a cabo este proyecto, se ha utilizado una metodología comunicativa y de tutoría entre iguales partiendo del aprendizaje-servicio. Se han diseñado e implementado, conjuntamente entre expertos, alumnado y mujer gitana, talleres destinados a trabajar la inserción laboral, las emociones y el estudio de los estereotipos y roles de género. Los resultados muestran la adquisición y desarrollo de competencias para la inclusión en el colectivo de mujeres gitanas, así como un aumento del nivel formativo, la motivación y el interés en el alumnado participante.
\end{abstract}

\section{KEYWORDS:}

Service-learning

Innovation

Gypsy woman

Students

Social justice

\begin{abstract}
:
This work shows the fundamental contributions of a Teaching Innovation Project funded by the University of Granada during the 2019/2020 academic year. Its dual purpose has made it possible to work on the training-social inclusion of gypsy women residing in the North Zone of the city of Granada and, on the other hand, to develop professional skills in students who are studying social education and pedagogy degrees. To this end, a collaborative work process has been put into practice in which gypsy women have participated together with professionals from social education and pedagogy, as well as students from those degrees. To carry out this project, a communication and peer tutoring methodology was used based on service-learning. Workshops have been designed, implemented and put into practice jointly between experts, students and gypsy women, aimed at working on labor insertion, emotions and the study of gender stereotypes and roles. The results show the acquisition and development of competences for inclusion in the group of Roma women, as well as an increase in the level of training, motivation and interest in the participating students.
\end{abstract}




\section{Introducción}

Una escuela inclusiva es aquella que está en movimiento, más que aquella que ha conseguido una determinada meta (Durán y Climent, 2017), es un proceso de identificación y minimización de los factores de exclusión, inherentes a las instituciones sociales, que ayuda por tanto al equilibrio y a la consecución de derechos fundamentales y de justicia social (Jiménez et al., 2017; Murillo y Hernández, 2014). La educación se convierte en una poderosa herramienta para luchar contra la exclusión como se afirma desde la UNESCO (1999):

\section{La educación puede ser un factor de cohesión social si procura transformar la diversidad en un factor positivo de entendimiento mutuo entre los individuos y los grupos humanos y al mismo tiempo evita ser (ella misma) un factor de exclusión social. (p. 59)}

Numerosas investigaciones (Camargo et al., 2021; Cárdenas-Rodríguez et al., 2019; Guichot-Reina, 2021; Jiménez y Fardella, 2015; Shaeffer, 2019) ofrecen un panorama general de lo que el fenómeno de la inmigración ha supuesto para las escuelas. En términos generales, sus hallazgos y discusiones advierten de la complejidad y urgencia de las problemáticas que la escolarización del alumnado inmigrante y perteneciente a minorías étnicas ha supuesto para el sistema educativo en su conjunto.

La educación inclusiva se constituye hoy en día como la opción más adecuada para afrontar el reto de educar a todos y todas por igual, ya que defiende los derechos humanos y hace suyos los principios de igualdad, equidad y justicia social (Ainscow et al., 2006; Costelo y Boyle, 2013; Santos et al., 2012).

La educación inclusiva exige una especial atención a la creación de una comunidad cálida y acogedora para todos los estudiantes del aula y la escuela (Sapon, 2013). Un currículum abierto y preparado para la diversidad, parte de un centro con una cultura escolar que admite las diferencias y las trata abiertamente. Un centro donde la exclusión y la marginación no tienen cabida (Arnaiz y Guirao, 2015; Sapón, 2013). Y donde se trabaje a partir de la colaboración y el consenso de los agentes implicados en el sistema educativo (Escarbajal et al., 2020).

Atendiendo a esta diversidad es necesario hablar del pueblo gitano, el cual lleva conviviendo con la sociedad española desde el siglo XV y su tamaño poblacional en nuestro país oscila entre 500.000 y 1.000.000 de personas. Los procesos de adaptación cultural a los que han sido sometidos vienen dados por una serie de pragmáticas y normativas, normalmente discriminatorias, que han ido construyendo el espacio donde hoy en día este pueblo se encuentra. Si atendemos a la variable género, y en concreto al perfil de la mujer gitana, podemos percatarnos de las desigualdades que durante toda la historia han tenido que afrontar por el mero hecho de ser mujer, aspecto que se ha ido reproduciendo y que tiene un reflejo actual en nuestra sociedad. Según Zugaza (2017), la exclusión del colectivo gitano tanto en términos económicos como de ausencia en lo público es aún un reto. Esta situación es más crítica para las mujeres gitanas, ya que les acecha la invisibilidad y el estigma de manera incuestionable. Sobre esta realidad radica la importancia del proyecto de innovación en el que se sustenta el presente artículo.

Un sistema institucional justo no puede, en ningún grado, victimizar (o premiar) a las personas por la suerte o desgracia en la que ha nacido, al contrario, debe tender activamente a contrarrestarlo. Una sociedad justa debe tender, en lo posible, a igualar a las personas en sus circunstancias, de tal modo que lo que ocurra con sus vidas quede bajo su propia responsabilidad. Por tanto, una institución básica como la educativa debe poner todos los medios para contrarrestar dichas situaciones de desventaja (Bolívar, 2012) fundamento del proyecto de innovación y base del trabajo con el alumnado de los grados de educación social y pedagogía.

Como consecuencia, el objetivo general del proyecto de innovación desarrollado se ha centrado en mejorar la realidad del colectivo mujer gitana en riesgo de exclusión debido al abandono temprano del sistema educativo obligatorio. Para ello se ha trabajado desde la cooperación y el apoyo competencial para la implementación en entornos desfavorecidos, a través del aprendizaje-servicio como metodología de trabajo inclusivo y ayuda a la justicia y cohesión social. El aprendizaje-servicio es considerado una práctica educativa innovadora que combina objetivos de aprendizaje curricular con objetivos de servicio a la comunidad, con la intención de mejorar las realidades donde se realiza el servicio, y que considera a quien recibe el mismo como un elemento central y no como un simple 
receptor de actos de caridad. El cual contribuye a una nueva definición del concepto de educación, en tanto que fomenta el aprendizaje significativo y revitaliza la dimensión ética y ciudadana de los procesos educativos (Aramburuzabala, 2013; Zarzuela y García, 2021; Zayas et al., 2018).

Una estrategia más que ayuda a la inclusión social consiste en utilizar metodologías cooperativas como la tutoría entre iguales, basado en la creación de parejas (derivada del rol respectivo de tutor o tutorado) con el objetivo de adquisición de competencias curriculares y utilizada en todos los niveles educativos (Durán y Vidal, 2004). La tutoría entre iguales constituye, en este sentido, un recurso pedagógico importante y cada vez más valorado en el contexto universitario. La justificación del aprendizaje entre pares reside en que los compañeros y compañeras pueden ser una fuente confiable y creíble de información, compartiendo experiencias similares, así como normas sociales, por lo tanto, están en mejor posición para proporcionar información relevante, significativa, explícita y honesta (Vargas y García, 2021).

El presente artículo describe la metodología empleada, una metodología cooperativa entre expertos y alumnado de los grados de educación social y pedagogía, para la elaboración de talleres y su implementación en entornos desfavorecidos, a través del trabajo entre iguales y la experiencia y orientación de las mujeres gitanas. Llevando a cabo una educación experiencial con un potencial de movimiento social transformador, enmarcado en el desarrollo de un proyecto de innovación de la Universidad de Granada.

\section{Inclusión y justicia social. Colectivos vulnerables}

Estudios actuales reconocen mayor brecha entre colectivos pertenecientes a contextos desfavorecidos (Cárdenas-Rodríguez et al., 2019), por lo que el desarrollo de propuestas inspiradas en principios de inclusión, multiculturalidad y justicia social cobran cada vez más relevancia (Jiménez et al., 2017). La educación inclusiva no es un estado, sino un proceso, se trata de un viaje que nunca acaba, se concibe como un proceso de progreso del centro, con la participación de toda la comunidad educativa (Ainscow, 2005).

Diferentes trabajos muestran el acuerdo unánime sobre la educación inclusiva pero en la actualidad una limitación de las discusiones sobre la inclusión versa sobre la incapacidad de reconocer que las políticas escolares son reflejo de valores de la sociedad más generales, y que las barreras para la educación inclusiva están por tanto profundamente arraigadas en la estructura social, política, económica e ideológica, llegando a ser un tema y una preocupación creciente de justicia social (Murillo y Hernández, 2014).

Desde esta concepción de escuela, hablar de inclusión es hablar de justicia y, parece lógico, que para construir una sociedad distributiva y honesta es necesario desarrollar modelos educativos equitativos que afronten con justicia los desequilibrios existentes en la misma (López Melero, 2011; Shaeffer, 2019).

La atención al alumnado que presenta cualquier tipo de vulnerabilidad, debe ser un tema central de cualquier comunidad educativa. Es necesario trabajar y resaltar la precisa adaptación del sistema y los centros educativos a las distintas diversidades coexistentes, realizando acciones preventivas y de mejora de la atención educativa para evitar que surjan las necesidades puesto que es el entorno el que no proporciona las oportunidades necesarias y no es responsabilidad del alumnado sino de las instituciones educativas.

En este sentido, tal como denomina Wehmeyer (2009), tres ideas merecen ser destacadas en la comprensión de lo que hoy en día se entiende por inclusión educativa. En primer lugar, el proceso de inclusión supone trasladar el foco de atención del "alumno" al "contexto". No son tan importantes las condiciones y características de los alumnos/as cuando la capacidad del centro educativo es de acoger, valorar y responder a las diversas necesidades que plantea el alumnado; capacidad que debe reflejarse en el pensamiento del profesorado, en las prácticas educativas y en los recursos personales y materiales disponibles. Hay que adaptar la propuesta educativa a un alumnado diverso, y no al revés, 
así como a la provisión de los apoyos que eventualmente un alumno pueda precisar (Arnaiz y Azorín, 2014).

En segundo lugar, la inclusión no debe restringirse al alumnado con condiciones personales de discapacidad; la inclusión tiene que ver con promover más y mejores oportunidades para todos los alumnos/as, y en particular para aquellos que por diversas razones (migratorias, culturales, sociales, de género, discapacidad) pueden estar en mayor riesgo de exclusión y fracaso (Muntaner, 2014).

Y, por último, la educación inclusiva, como se ha mencionado antes, es un proceso (Ainscow, 2005), un proceso de mejora del centro, con la participación de toda la comunidad educativa. De identificación y minimización de los factores de exclusión, inherentes a las instituciones sociales, que ayuda por tanto al equilibrio y a la consecución de derechos fundamentales y de justicia social (Murillo y Hernández, 2014; Quintanal et al., 2016). Desde esta perspectiva carece de sentido hablar o dividir las aulas o los centros en inclusivos o no inclusivos. La inclusión no es una meta a la que se llega, sino un compromiso sostenido a favor de mejores condiciones y oportunidades para todo el alumnado (Ainscow, 2005; Durán y Climent, 2017). Estudios recientes demuestran que dentro del ámbito organizativo de centro y aula la utilización de grupos heterogéneos conlleva mayores niveles de inclusión. Así como la participación de familias en los grupos heterogéneos para enriquecer y favorecer el aprendizaje (Escarbajal et al., 2020).

Las escuelas que trabajan por la justicia social son escuelas con temáticas y funcionamiento justo, generadoras y promotoras de colaboración entre escuela y familia con una clara finalidad de expansión del capital social de los estudiantes (Jiménez et al., 2017; Murillo y Hernández, 2014). Son escuelas capaces de llevar a cabo un proceso de Redistribución, Reconocimiento y Representación. La base está en desarrollar principios de justicia que permitan corregir la privación de recursos y que estos sean mejor distribuidos en función de los distintos intereses (Belavi y Murillo, 2020; Hidalgo y Murillo, 2016; Murillo et al., 2014).

Los centros educativos que trabajan por y para la inclusión, para la consecución real de derechos, deben ayudar a la equitativa distribución de la educación para todos y todas. Como estructura básica de la sociedad, debe ayudar a las libertades, lo que se relaciona directamente con la justicia como equidad y al principio de la diferencia, que hace referencia a la estructura social y la contribución que debe hacer ésta a la mejora de los miembros más desfavorecidos de la sociedad (Vences, 2020).

Ofrecer una respuesta educativa que favorezca la inclusión implica llevar a cabo mecanismos de coordinación y colaboración docente que ayuden a caminar en una misma dirección; así como la utilización de metodologías variadas que faciliten el acceso al aprendizaje, entre ellas: agrupamientos heterogéneos, adecuación de actividades o uso de diversos procedimientos para la evaluación (Escarbajal et al., 2019, 2020; Inostroza-Barahona, 2021). La justicia social tendrá que enfatizar en las prácticas sociales y la calidad de las formas de reconocimiento ante cualquier marginación o exclusión (Montané, 2015).

En los últimos 20 años hemos vivido una situación de acercamiento y progresiva cooperación entre la institución escolar, sus pedagogías y la pedagogía social. En este contexto, se viene tendiendo a convertir los centros escolares en territorios de acción, también de educadores y educadoras sociales, replanteando con ello ciertos esquemas propios de la cultura escolar y, siempre de manera complementaria, de la propia función docente y educativa (Vila et al., 2020).

\section{Cohesión e inclusión como eje innovador}

\subsection{Justificación del proyecto: Fundamentación normativa}

Tras la revisión fundamental de inclusión y justicia social se asientan los pilares y la justificación del trabajo de innovación realizado con mujer gitana en la Zona Norte de la ciudad de Granada. El fundamento legal del trabajo parte del principio de inclusión recogido en sus inicios en el título II de la Ley Orgánica de Educación (LOE), donde señala la equidad como garantía de la igualdad de oportunida- 
des y la inclusión educativa de derechos y oportunidades. Todo ello incluido en la Ley Orgánica 2013 para la mejora de la calidad educativa (LOMCE), donde se recoge que "La atención a los alumnos/as que presentan cualquier tipo de vulnerabilidad, debe ser un tema central de cualquier comunidad educativa. Así como la clasificación del alumnado con NEAE (necesidades específicas de apoyo educativo) LOMLOE (2020):
Corresponde a las Administraciones educativas asegurar los recursos necesarios para que los alumnos y alumnas que requieran una atención educativa diferente a la ordinaria, por presentar necesidades educativas especiales, por retraso madurativo, por trastornos del desarrollo del lenguaje y la comunicación, por trastornos de atención o de aprendiza- je, por desconocimiento grave de la lengua de aprendizaje, por encontrarse en situación de vulnerabilidad socioeducativa, por sus altas capacidades intelectuales, por haberse incorporado tarde al sistema educativo o por condiciones personales o de historia escolar, puedan alcanzar el máximo desarrollo posible de sus capacidades personales y, en todo caso, los objetivos establecidos con carácter general para todo el alumnado. (Artículo 2)

La LOE en su capítulo II, señala la compensación de las desigualdades donde se menciona la obligación de la administración de desarrollar actuaciones de carácter compensatorio, para eliminar desigualdades generadas por factores sociales, económicos, étnicos o de otra índole.

Hacer mención a la LOMLOE donde se menciona que, para impulsar la equidad del sistema educativo, se fortalecen en el capítulo segundo de este título los objetivos y las actuaciones que deben llevar a cabo las Administraciones educativas ante las personas, grupos, entornos sociales y ámbitos territoriales que se encuentren en situación de vulnerabilidad socioeducativa y cultural, a fin de eliminar las barreras que limitan su acceso, presencia, participación y aprendizaje. Con ello se quieren asegurar los ajustes razonables en función de las necesidades individuales y prestar el apoyo necesario para fomentar su máximo desarrollo educativo y social, de manera que todos y todas puedan acceder a una educación inclusiva, en igualdad de condiciones con los demás.

Se destaca la Estrategia nacional para la inclusión social de la población gitana en España 2012-2020 (Ministerio de Sanidad, Servicios Sociales e Igualdad, 2014), donde se recoge la estrategia a nivel educativo, de empleo, salud y vivienda propuesta para la mejora del colectivo gitano. El Informe 2019 sobre el estado del sistema educativo español ${ }^{1}$, menciona la necesidad de mejorar e intervenir con el colectivo de mujer gitana dentro de las políticas para la igualdad de oportunidades. Respaldado por la Ley $9 / 2018^{2}$, de 8 de octubre, de modificación de la Ley 12/2007, de 26 de noviembre, para la promoción de la igualdad de género en Andalucía donde se recoge que «la igualdad de género es parte integrante de los derechos humanos y es un requisito fundamental de la democracia»; reconocía que «el estatus legal de las mujeres ha mejorado con el tiempo, pero, pasados veinte años desde la Declaración sobre la igualdad de mujeres y hombres ${ }^{3}$ todavía es un reto para los Estados miembros salvar la distancia entre la igualdad legal y la real», y reafirmaba que «una auténtica democracia debe aprovechar las competencias, habilidades y creatividad de las mujeres y de los hombres para construir una sociedad con mayor calidad de vida para todas las personas y que respete los principios en los que se funda el Consejo de Europa».

Autores como López Rodríguez y Sanz Hernández (2017) reconocen cómo "las mujeres gitanas, en su ir y venir entre el adentro y el afuera de su cotidianidad -gitanidad-, cargan y arrastran una mochila de identidad que determina su peso en la infraestructura relacional étnica” (p. 45). Existe, por tanto, una clara necesidad de establecer estrategias para favorecer el proceso educativo de este colectivo, así como su inclusión social y educativa. El desarrollo de propuestas inspiradas en principios de inclu-

1 Consejo Escolar del Estado. (2019). Informe 2019 sobre el estado del sistema educativo. Curso 2017-2018. http://www. educacionyfp.gob.es/mc/cee/publicaciones/informes-del-sistema-educativo/informe-2019.html

2 https://www.boe.es/diario_boe/txt.php?id=BOE-A-2018-15239

3 https://www.consilium.europa.eu/media/20602/1988_junio_-_hannover_es_.pdf 
sión, multiculturalidad y justicia social cobran cada vez más relevancia (Guichot-Reina, 2021; Jiménez et al., 2017).

La educación intercultural requiere la construcción de una ética pública y respuestas institucionales con base del interculturalismo, construidas a partir de principios capitales como son la igualdad de derechos, la equidad, entendiendo que el alumnado no debe enfrentar barreras en su aprendizaje (Terrón et al., 2017).

\subsection{Justificación del proyecto: Fundamentación psicopedagógica}

Dentro de los perfiles del pedagogo/a y del educador/a social existe una clara necesidad de apoyar la figura de ambos en contextos de educación formal. No solo a nivel de la calle Molino Nuevo, con un índice de exclusión y marginación social alto, sino también por las múltiples facetas a las que ambos profesionales deben responder en todos los ámbitos. El apoyo y el trabajo conjunto en ámbitos formales es una necesidad cada vez más acuciante en educación, debido a los altos índices de abandono escolar de colectivos desfavorecidos y en este caso en concreto, por la situación de la mujer y de la mujer gitana en la sociedad y en el contexto actual. La intervención pedagógica y social es fundamental en contextos formales con población vulnerable, y el apoyo de todos los agentes implicados en el contexto educativo es cada vez más notable. De ahí la necesidad de la formación desde la universidad, no solo en las competencias recogidas en el Libro Blanco del pedagogo/a y del educador/a social, sino en el apoyo mutuo de ambos profesionales debido a los cambios sociales que se están produciendo en la actualidad.

Los y las profesionales de la educación social coinciden en destacar como debilidades de la profesión las carencias formativas con un peso del $30 \%$, ya que demandan más formación teórica-práctica, pero sobre todo específica y focalizada en las áreas de acción socioeducativa (Eslava-Suanes et al., 2018; Terrón et al., 2017; Vila et al., 2020).

Diversas investigaciones enfatizan la necesidad de la intervención socioeducativa en realidades multiculturales y holísticas las cuales demandan equipos de trabajo multidisciplinares, con personas con distintos perfiles profesionales, entre los que se encuentran los/las educadores/as sociales (Moliner et al., 2016; Pérez de Guzmán et al., 2020; Terrón et al., 2017; Viana-Orta et al., 2019; Vilar, 2018).

\subsection{Diagnóstico de la realidad}

El proyecto parte del programa de trabajo con mujer gitana denominado "Atrévete" llevado a cabo en el Centro Luisa de Marillac de la Zona Norte de la ciudad de Granada y puesto en marcha desde la Delegación de Educación con el objetivo de erradicar el fracaso escolar y el abandono de los estudios de Educación Secundaria Obligatoria, y más concretamente de las alumnas gitanas embarazadas tras terminar los estudios de Educación Primaria con abandono escolar debido a su situación. Este proyecto, llevado a cabo en varios centros de la provincia, ayuda a las jóvenes gitanas de entre 12 y 16 años con un alto índice de absentismo escolar y posterior abandono de los estudios por su situación familiar. Acuden al centro Luisa de Marillac por las tardes donde el profesorado del mismo les imparte docencia, este trabajo se realiza en conexión con los Institutos de la Zona Norte para conseguir ser tituladas en Educación Secundaria Obligatoria y tener más posibilidades de encontrar un empleo. Haciendo referencia al contexto universitario al que va referido el artículo, se parte de una necesidad real de conexión y apoyo mutuo entre el trabajo conjunto de educadores/as sociales y pedagogos/as en entornos formales de enseñanza, tanto desde las competencias específicas dentro de los estudios de grado, como en la situación real en el contexto descrito, justificado en el marco inicial. La implicación de ambos profesionales es fundamental para el éxito de la cohesión social y de la inclusión socioeducativa de colectivos marginales. Las competencias descritas en el Libro Blanco, tanto para educadores/as como para pedagogos/as, enfatizan las funciones de coordinación y trabajo en equipo en diferentes contextos. La formación del alumnado en la universidad en cooperación y apoyo profesional debe ir encaminado a la mejora de las titulaciones en ambos aspectos, colaboración e inclusión socioeducativa. 


\subsection{Objetivos}

Desarrollar un proyecto de innovación para el desarrollo de trabajo colaborativo entre mujer gitana, educadores/as sociales y pedagogos/as, con la finalidad de mejorar las competencias profesionales del alumnado y la formación-inclusión social de la mujer gitana. Del mismo modo, preparar al alumnado de los grados de educación social y pedagogía, en el trabajo cooperativo y en la metodología comunicativa, teniendo como base el aprendizaje-servicio, para trabajar de forma conjunta con colectivos desfavorecidos y en concreto mejorar la realidad de la mujer gitana en la Calle Molino Nuevo (zona norte de Granada).

\subsection{Metodología general dentro del aula y plan de trabajo}

- Metodología comunicativa a través de interpretaciones y reflexiones entre los miembros del grupo y del diálogo igualitario. Realizado desde los seminarios iniciales de formación del alumnado de los grados con los expertos, así como en el seminario de intercambio de información con las chicas gitanas que visitaron la universidad.

- Tutorías entre iguales entre las alumnas que han superado los estudios de la ESO y las alumnas del plan actual. Apoyo y guía de las alumnas gitanas que ya han promocionado y las mujeres gitanas en formación dentro del Proyecto. Así como entre los tres alumnos del grado de Pedagogía voluntarios en el proyecto y los/as alumnos/as del grado de Educación Social.

- Método del aprendizaje-servicio que permite la unión del aprendizaje con el compromiso social. Estrategia llevada a cabo desde las aportaciones del trabajo de los expertos en la zona norte a través de la formación del alumnado de los grados y la posterior salida a las intervenciones en el centro Luisa de Marillac con mujer gitana, con la finalidad de mejorar la situación del colectivo.

El plan de trabajo se presentó a través de tres fases:

\section{Fase 1. Formación}

Formación del alumnado de los grados sobre el colectivo vulnerable a través de seminarios con mujeres de etnia gitana que superaron el proyecto "Atrévete" y que habían obtenido el título de la ESO, donde narraron su experiencia y sus necesidades, dialogando con el alumnado del grado. Estas mismas alumnas compartieron espacio y seminario con las alumnas del proyecto actual en curso con la finalidad de dialogar y detectar necesidades del colectivo.

Formación en seminario inicial con los expertos de las temáticas de los talleres. Durante seminarios de clase el/la experto/a en las temáticas formó a los estudiantes para la intervención posterior, aportando estrategias para la elaboración de las actividades.

\section{Fase 2. Intervención}

Puesta en práctica del trabajo de campo, el cual consistió en una formación inicial desarrollada en los seminarios anteriormente descrito y la realización y ejecución de los diferentes talleres entre el alumnado de educación social y pedagogía a las mujeres gitanas del Proyecto Atrévete. Dicha intervención se realizó en el Centro Luisa de Marillac, en horario de tarde y dentro de un entorno formal de enseñanza-aprendizaje.

Los talleres desarrollados se centraron en:

- Orientación laboral para fomentar la inserción socio laboral, igualdad y búsqueda de empleo.

- Empleo de las TIC para reducir la brecha digital.

- Las emociones para aprender a gestionarlas.

- Análisis de los estereotipos y roles de género asociados a la cultura gitana. 
Participantes:

- 4 profesores/as de la Facultad de Ciencias de la Educación de la Universidad de Granada.

- 4 expertos/as para la elaboración de los talleres (educadores/as sociales en activo y pedagogos/as)

- 7 mujeres gitanas del proyecto en curso. 10 mujeres gitanas del proyecto Atrévete finalizado.

- 59 alumnos/as del grado de Educación Social y Pedagogía. Asignatura: Orientación profesional en educación social: reflexión ética y deontológica y tres alumnos/as de cuarto de Pedagogía pertenecientes al proyecto.

Cuadro 1

Plan de trabajo

Seminario formación al alumnado del Grado dentro de la Universidad. Un seminario por semana en gran grupo

Semana del 14-29 de septiembre 2019: impartido Seminarios teóricos y de formación en intervención sobre la por profesores-miembros del equipo de trabajo temática objeto de innovación a los alumnos del Grado.

Semana del 30 septiembre-06 octubre de 2019: Expertos del Colegio (pedagogos y educadores/as sociales de la zona norte)

Semana del 7-13 octubre de 2019: visita de las alumnas gitanas del proyecto al aula de la Universidad

Semana del 14-20 octubre de 2019

Semana del 21-27 octubre de 2019

Semana del 28 octubre-3 noviembre de 2019

Semana del 4-10 noviembre de 2019
Formación práctica sobre la zona norte y colectivo gitano a los alumnos/as del Grado de E.S y alumnado de Pedagogía propio del proyecto. Visita al centro Luisa de Marillac. Detección de necesidades.

Formación desde la tutoría entre iguales y el diálogo igualitario. Detección de necesidades.

Seminario Orientación laboral para fomentar la inserción socio-laboral, igualdad y búsqueda de empleo. Experto en educación social y educador de calle.

Seminario Experta en roles de Género y estereotipos. Educadora Social-Pedagoga.

Seminario de emociones.

Seminario en TIC y brecha digital.

Talleres de intervención (salida de los alumnos/as del Grado al centro, acompañados por el docente y el experto del seminario) de dos a tres grupos por semana, 30-45 minutos por taller. División del grupo clase en grupos de trabajo (grupos de 5-6 alumnos/as)

Segunda del 11-17 noviembre de 2019

Semana del 18-24 noviembre de 2019

Semana del 25-30 noviembre de 2019

Semana de 2-15 diciembre de 2019

Semana de 16-23 diciembre de 2019 y 13-19 enero de 2020
Taller de emociones.

Taller de emociones.

Taller de inserción laboral en el centro Luisa de Marillac.

Taller de estereotipos de género.

Talleres TIC.

Fase 3. Evaluación

Semana del 20-26 de enero de 2020

Evaluación de resultados y recogida de información: Entrevista inicial y final a alumnas del proyecto Atrévete y al alumnado del Grado (Pedagogía y Educación Social)

Nota. Elaboración propia

La evaluación parte de un enfoque cualitativo, el cual nos permite describir, interpretar y reflexionar sobre la información obtenida mediante el proceso de investigación. Se plantea el análisis de una realidad social contextualizada desde la perspectiva de las mujeres gitanas y del alumnado de grado, desarrollado un estudio cualitativo de corte descriptivo y hermenéutico, caracterizado por un pro- 
ceso deductivo-inductivo. El instrumento de recogida de información utilizado ha sido la entrevista semiestructurada diseñada ad hoc y validada a través de un juicio de expertos. Las entrevistas se grabaron en audio, bajo previo consentimiento de cada uno de los entrevistados, para posteriormente, realizar un análisis de contenido, utilizando el programa Atlas.ti versión 8, con el fin de organizar, reducir y analizar la información recabada.

Ejemplo y descripción de seminario: Uno de los seminarios puestos en práctica fue el denominado "El arte de educar a un dragón. El educador social en la frontera”.

El seminario se desarrolló durante una hora para aproximar al alumnado de educación social y pedagogía hacia una visión real y práctica del rol que cada profesional tiene en realidades socioeducativas que se sitúan en la frontera social, en realidades de vulnerabilidad y de riesgo de exclusión social. Este seminario-reflexión fue realizado por un experto "educador social de calle" con una amplia experiencia en la educación social y consistió en una primera visión práctica sobre los rasgos identitarios que deberían tener con estos destinatarios de realidades sociales desfavorecidas, en especial con la mujer gitana. El seminario práctico se dividió en tres episodios, los cuales se describen a continuación:

\section{Episodio 1. Los dragones existen}

A través de este primer episodio los participantes se sumergieron en el trabajo y conocimiento de los perfiles de menores y familias en riesgo de exclusión social; se abordaron algunos casos concretos y se presentó la realidad de compensación de desigualdades en educación. Se trabajaron las nuevas bolsas de pobreza juvenil y los grupos de radicalización de violenta juvenil.

Figura 1

Reflexión inicial por grupos

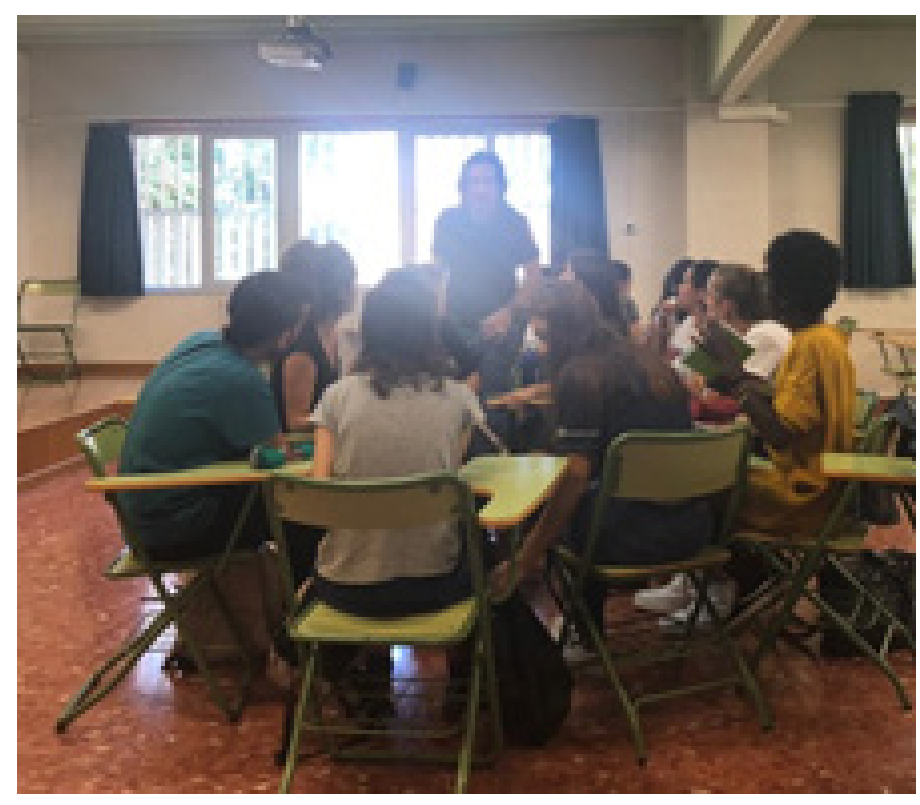

\section{Episodio 2. La espada del valor}

Se explicaron algunas estrategias de intervención del educador/a social y el pedagogo/a en estos paisajes de exclusión. Se subrayó la importancia del ser educador/a y pedagogo/a así como del buen hacer como profesionales de la educación. Se incidió sobre la necesidad imperiosa de cambiar y reenfocar la intervención educativa.

\section{Episodio 3. Fronteras de intervención}

Se reflexionó sobre la importancia de la vocación de ambas profesiones; aportando un mapa de realidades sociales emergentes donde el educador/a y el pedagogo/a pueden trabajar, desarrollando la motivación e ilusión para intervenir en estas realidades socioeducativas. 
Figura 2

Ejemplo de actividad reflexiva

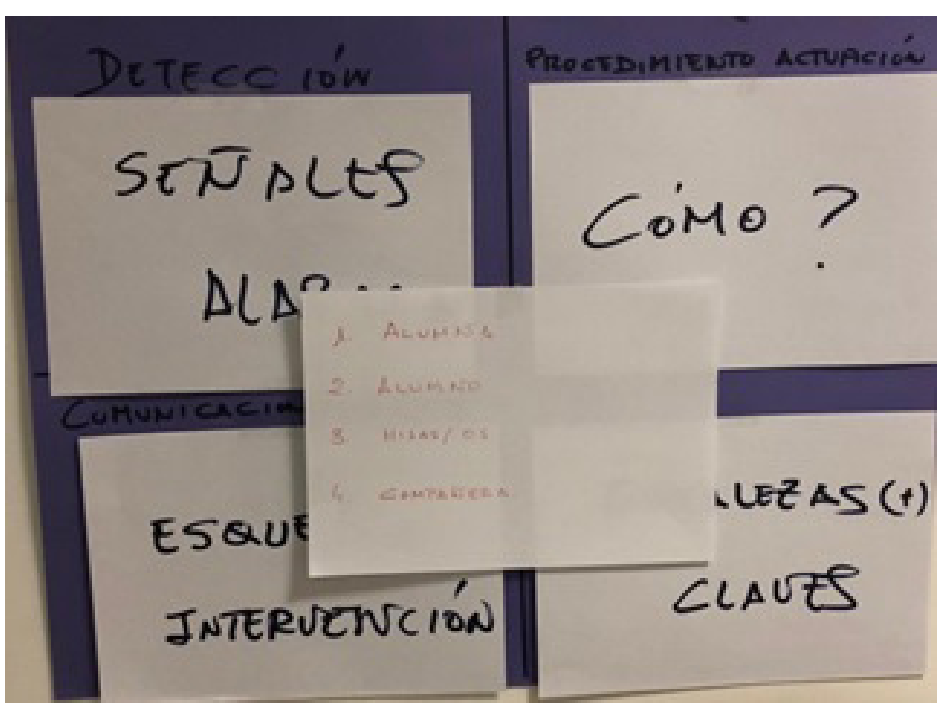

\section{Resultados}

Partimos de dos aspectos esenciales, el primero es poder dar voz a la mujer perteneciente a colectivos desfavorecidos ya que la discriminación de la mujer gitana, por ser mujer, por ser gitana y por falta de estudios es cada vez más una necesidad real para ayudar a la cohesión social, faceta que se ha llevado a cabo en la universidad a través de la formación y la inserción laboral, las emociones y el análisis de los estereotipos y roles de género asociados a la cultura gitana. Un segundo aspecto a resaltar ha consistido en conectar a los alumnos/as de los grados de educación social y pedagogía con la realidad, el aprendizaje-servicio a la comunidad y el trabajo colaborativo de ambos colectivos. La finalidad de la misma ha mejorado la formación de los estudiantes de grado con el colectivo de mujer en riesgo de exclusión.

Dentro de los perfiles del pedagogo/a y del educador/a social se ha apoyado la figura de ambos colectivos en contextos de educación formal, no solo a nivel de la calle Molino Nuevo, con un índice de exclusión y marginación social alto, sino también por las múltiples facetas a las que ambos profesionales deben responder en todos los ámbitos. El apoyo y el trabajo conjunto en ámbitos formales es una necesidad cada vez más acuciante en educación, debido a los altos índices de abandono escolar de colectivos desfavorecidos y en este caso en concreto, por la situación de la mujer y de la mujer gitana en la sociedad y en el contexto actual. La intervención pedagógica ha sido fundamental en este contexto formal con población vulnerable, y el apoyo de todos los agentes implicados en el contexto educativo ha sido muy notable. De ahí la necesidad de la formación desde la universidad, no solo en las competencias recogidas en el Libro Blanco del pedagogo/a y del educador/a social, sino en el apoyo mutuo de ambos colectivos debido a los cambios sociales que se están produciendo en la actualidad.

La evaluación del proyecto se realizó en tres momentos, una evaluación inicial en la cual el profesorado detectó una serie de necesidades en el contexto a trabajar mediante la observación participante y el diálogo conjunto con los expertos que trabajan en el proyecto "Atrévete”, junto a la realización de una entrevista por parte del alumnado para conocer la formación en torno al colectivo o cómo se podría llevar a cabo una autoformación o formación en la Universidad acerca de esta temática. Una evaluación procesual a través de encuentros reflexivos entre el alumnado y las mujeres gitanas, previos a la realización de cada uno de los talleres y una vez finalizados para compartir experiencias, aprendizaje y necesidades. Por último, una evaluación final en ambos colectivos a través de entrevistas para conocer la evaluación 
general de la puesta en práctica del proyecto. Se destacan algunos extractos de las evaluaciones finales realizadas, del alumnado ${ }^{4}$ :

Esta oportunidad es muy importante para mejorar mi formación en la universidad, he de decir que es la primera vez que puedo tener un contacto tan estrecho con la realidad. (I19:9-28)

Al principio creía que no sería capaz de poder desarrollar mi práctica profesional con las mujeres gitanas pero con la ayuda y la formación de los expertos he aprendido mucho, también he aprendido a confiar más en mí y en mi trabajo. (I20-30-45)

Sí, creo necesaria una formación ya que es un colectivo olvidado por la sociedad y la cual tienen muchos prejuicios hacia ellos, y tener información e indagar sobre su historia, su cultura, sus tradiciones me parece interesante que como profesional se me brinde la oportunidad de formarme y conocer a este colectivo involucrado. (I2-20-30)

A lo largo de la carrera no he recibido ninguna formación sobre este colectivo. No hay ninguna formación en la cual se nos enseñe como poder trabajar con ellos y hacer visible su etnia y que tengan un buen lugar en la sociedad como todo el mundo. (I15-45-40)

Se podría llevar a cabo una formación en la universidad mediante algún proyecto que esté relacionado con el colectivo y dejarnos acudir para poder formarnos y tener conocimientos de ello. (I6-48-60)

He de decir que este proyecto me ha sorprendido gratamente, es la primera vez que he podido conocer y trabajar con el colectivo de la mujer gitana. (I18-26-35)

Estoy feliz por cómo los profesores, los expertos y las mujeres me han dado la posibilidad de acercarme de manera profesional y personal a este colectivo. De las mujeres gitanas. (I4-3549)

Como mujeres gitanas estamos realmente orgullosas de poder pisar y venir a la universidad para contar nuestra humilde historia. (I50-28-39)

A través de la realización de estos talleres he aprendido mucho, sobre todo en el de buscar trabajo ya que desde pequeña se me han inculcado unos valores del hogar, gracias al tallery a los profesionales conozco otros modos diferentes de vivir. (I52-29-60)

Es la primera vez que vemos a alumnos de la universidad venir a nuestro barrio y eso creo que es bueno. (I53-25-23)

Espero que este tipo de proyecto se realice más veces, he aprendido mucho, a veces he sentido que estaba en la universidad como todos ellos. (I48-33-50)

Me acuerdo mucho de mis allegadas, me gustaría que hubiesen tenido la misma oportunidad que yo de aprendery poder conocer la universidad. (I49-43-48)

La formación que me han dado me va a ayudar a poder desarrollarme como persona, me faltaba dicha formación al respecto y con el desarrollo de los talleres creo que puedo desempeñar algunas funciones que antes no sabía. (I53-46-60)

\section{Discusión y conclusiones}

Para terminar, queremos destacar la necesidad de incrementar y mejorar las iniciativas dirigidas a construir estrategias orientadas a la eliminación de la discriminación de la desigualdad que afecta a la mujer gitana en España, es por ello, que es muy necesario mejorar la formación de personas que trabajan especialmente en estos campos (Arza y Laparra, 2014). Así como el desarrollo de una formación universitaria de calidad, como el proyecto que se presenta, el cual permite la capacitación de profesionales de la edu-

4 Informante 19, Documento 9, Línea 28. 
cación para la gestión de programas de intervención con la comunidad gitana, y en concreto, con la mujer gitana (Eslava-Suanes et al., 2018; Pérez de Guzmán et al., 2020).

Abrir la universidad al contexto supone incluir la perspectiva de la mujer gitana compartiendo espacios educativos, estrategias y talleres que estimulen la inserción del colectivo en la sociedad, trabajando los prejuicios de los implicados (Márquez y Padua, 2016). Y enfrentando al alumnado a su propia formación, carencias y necesidades utilizando para ello estrategias como la tutoría entre iguales (Vargas y García, 2021; Zarzuela y García, 2021). Este proyecto de innovación ha supuesto un espacio de formación crítica mejorando la motivación y participación de los alumnos/as de grado y de las alumnas gitanas. Todo ello implicó la formación de profesionales capaces de convivir en una sociedad cada vez más diversa, realizando talleres que favorezcan la creación de ambientes democráticos, bajo el prisma de la justicia social, siendo un concepto dinámico, que se encuentra en constante mejora y reflexión, indagando en la construcción de necesidades más justas e igualitarias (Mujica et al., 2018). Bajo el paraguas de la herramienta "aprendizaje-servicio", se ha comprobado que los estudiantes aprenden mientras actúan sobre las diversas necesidades del entorno con el objetivo de transformar la realidad mediante acciones de mejora, al tiempo que reflexionan de forma estructurada sobre la experiencia vivida (Aramburuzabala, 2013). Se ha comprobado y apoyado con los resultados aspectos como los que señalan Zayas y otros (2018). argumentando a favor de la necesidad de implementar proyectos de Aprendizaje Servicio en el espacio de Educación Superior, como enfoque pedagógico favorecedor de la ciudadanía democrática y crítica, la participación cívica y sin duda la formación de una ética profesional arraigada en el concepto de bien común.

Este tipo de aprendizaje implica dejar atrás, en cierta medida, algunas pedagogías tradicionales y dar un paso hacia una revolución en los procesos de enseñanza-aprendizaje, donde el estudiante es el protagonista de su propio aprendizaje, a partir de las experiencias vividas ante situaciones y problemáticas reales de su entorno más próximo (Ruiz-Corbella y García-Gutiérrez, 2019). La tutoría entre iguales (mujeres gitanas de los dos cursos y el alumnado del Grado) ha proporcionado una experiencia extra curricular, que ha ayudado en la valoración de la opinión del otro, sus necesidades y problemáticas más acuciantes.

Como principales limitaciones destacamos la falta de formación en trabajo cooperativo entre los alumnos/as de ambos grados, así como la inexperiencia del alumnado en trabajo de campo. A esto hay que sumarle el tiempo limitado para la implementación de los talleres, el número masivo de alumnos/as en el aula al desarrollar las actividades con las alumnas de etnia gitana, lo que ayudó entre otras cosas a la creatividad de los equipos de trabajo.

Entre las proyecciones de trabajo futuras incluimos la intención de solicitar un segundo proyecto de innovación de tipo avanzado que nos permita seguir trabajando en esta línea de intervención y en la mejora de las limitaciones encontradas.

\section{Agradecimientos}

Financiación obtenida por el Vicerrectorado de Calidad, Innovación y Prospectiva de la Universidad de Granada. Convocatoria Proyectos de Innovación Docente. Código: 19-20. Título: Mujer gitana: cohesión e inclusión socioeducativa. Trabajo cooperativo desde la educación social y la pedagogía.

\section{Referencias}

Ainscow, M. (2005). La mejora de la escuela inclusiva. Cuadernos de Pedagogía, 349, 78-83.

Ainscow, M., Booth, T. y Dyson, A. (2006). Improving schools, developing inclusion. Routledge. https://doi.org/10.4324/9780203967157

Aramburuzabala, P. (2013). Aprendizaje-servicio: Una herramienta para educar desde y para la justicia social. Revista Internacional de Educación para la Justicia Social, 2(2), 5-11

Arnaiz, P. y Azorín, C. (2014). Autoevaluación docente para la mejora de los procesos educativos en las escuelas que caminan hacia la inclusión. Revista Colombiana de Educación, 67, 227-245.

https://doi.org/10.17227/0120391.67rce227.245 
Arnaiz, P. y Guirao, J. M. (2015). La autoevaluación de centros en España para la atención a la diversidad desde una perspectiva inclusiva: ACADI. Revista Electrónica Interuniversitaria de Formación del Profesorado, 18(1), 45-101. https://doi.org/10.6018/reifop.18.1.214341

Arza, J. y Laparra, M. (2014). Una experiencia de formación universitaria para intensificar un cambio social: La comunidad gitana como protagonista. Revista Número, 30, 171-178.

Belavi, G. y Murillo, F. J. (2020). Democracia y justicia social en las escuelas: Dimensiones para pensar y mejorar la práctica educativa. REICE. Revista Iberoamericana sobre Calidad, Eficacia y Cambio en Educación, 18(3), 5-28. https://doi.org/10.15366/reice2020.18.3.001

Bolívar, A. (2012). Justicia social y equidad escolar. Una revisión actual. Revista Internacional de Educación para la Justicia Social, 1(1), 9-45.

Camargo, C. D. B., Fernández, A. H. y Cobo, M. O. (2021). Análisis a través de modelaje estructural de la relación entre prácticas docentes, pluriculturalidad e inclusión educativa. Revista Electrónica Interuniversitaria de Formación del Profesorado, 24(1), 87-99. https://doi.org/10.6018/reifop.436051

Cárdenas-Rodríguez, R., Terrón-Caro., T. y Monreal-Gimeno, M. C. (2019). Educación primaria y alumnas gitanas. Análisis de las barreras sociales en contextos de exclusión. Revista de Investigación Educativa, 37(1), 75-91. https://doi.org/10.6018/rie.37.1.326221

Costelo, S. y Boyle, C. (2013). Pre-service secondary teachers' attitudes Towards inclusive education. Australian Journal of Teacher Education, 38(4), 129-143. https://doi.org/10.14221/ajte.2013v38n4.8

Durán, G. y Climent, G. (2017). La formación del profesorado para la educación inclusiva: Un proceso de desarrollo profesional y de mejora de los centros para atender la diversidad. Revista Nacional e Internacional de Educación Inclusiva, 9(1), 18-26.

Durán, D. y Vidal, V. (2004). Tutoría entre iguales. De la teoría a la práctica. Graó.

Escarbajal, A., Corbalán, P. y Orteso, P. (2020). Análisis de la inclusión educativa en contextos vulnerables. Revista Colombiana de Educación, 78, 361-382. http://doi.org/10.17227/rce.num78-6776

Eslava-Suanes, M. D., González-López, I. y De León-Huertas, C. (2018). La identidad profesional del educador social a través de su perfil competencial. Ediciones Universidad de Salamanca, 19(1), 53-76.

Guichot-Reina, V. (2021). Educación, justicia social y multiculturalismo: Teoría y práctica en el aula. Teoría de la Educación, 33(1), 173-196. https://doi.org/10.14201/teri.22984

Hidalgo, N. y Murillo, F. J. (2016). Evaluación de estudiantes para la justicia social. Propuesta de un modelo. Revista Internacional de Educación para la Justicia Social, 5(2), 159-179. https://doi.org/10.15366/riejs2016.5.2.008

Inostroza-Barahona, C. (2021). Significados docentes sobre currículum, educación inclusiva y justicia social. Revista Saberes Educativos, 6, 200-211. https://doi.org/10.5354/2452-5014.2021.60716

Jiménez, F. y Fardella, K. (2015). Diversidad y rol de la escuela: discursos del profesorado en contextos educativos multiculturales en clave migratoria. Revista Mexicana de Investigación Educativa, 20(65), 419-441.

Jiménez, F., Lalueza, J. L. y Fardella, K. (2017). Aprendizajes, inclusión y justicia social en entornos educativos multiculturales. Revista Electrónica de Investigación Educativa, 19(3), 10-23.

https://doi.org/10.24320/redie.2017.19.3.830

López Melero, M. (2011). Barreas que impiden la escuela inclusiva y algunas estrategias para construir una escuela sin exclusiones. Innovación Educativa, 21, 37-54.

López Rodríguez, M. E. y Sanz Hernández, A. (2017). Reflexión, acción, decisión: Trayectorias en la construcción de la identidad de género en el patriarcado gitano. Empiria. Revista de Metodología de Ciencias Sociales, 38, 41-62. https://doi.org/empiria.38.2018.19705

Márquez, J. M. y Padua, D. (2016). Comunidad gitana y educación pública. La necesidad de construir un proyecto social y educativo compartido. Revista Interuniversitaria de Formación del Profesorado, 85, 91-101.

Ministerio de Sanidad, Servicios Sociales e Igualdad. (2014). Estrategia nacional para la inclusión social de la población gitana en España 2012-2020. Ministerio de Sanidad, Servicios Sociales e Igualdad 
Moliner, O. Sales, A. y Escobedo, P. (2016). Posibilidades y limitaciones de generar cultura de centro desde las prácticas compartidas de aula: El caso de la educación intercultural inclusiva. Estudios sobre Educación, 30, 51-70. https://doi.org/10.15581/004.30.51-70

Montané, A. (2015). Justicia Social y Educación. Revista de Educación Social, 20, 92-113.

Mujica, F., Inostroza, C. y Orellana, N. (2018). Educar las emociones con un sentido pedagógico: Un aporte a la justicia social. Revista Internacional de Educación para la Justicia Social, 7(2), 113-127. https://doi.org/10.15366/riejs2018.7.2.007

Muntaner, J. (2014). Prácticas inclusivas en el aula ordinaria. Revista Nacional e Internacional de Educación Inclusiva, $7(1), 63-79$.

Murillo, F. J. y Hernández, R. (2014). Liderando escuelas justas para la justicia social. Revista Internacional de Educación para la Justicia Social, 3(2), 13-32.

Murillo, F. J., Hernández, R., Hidalgo, N. y Martínez-Garrido, C. (2014). Elaboración y evaluación psicométrica de la escala de actitudes hacia la justicia social en educación. Revista Internacional de Educación para la Justicia Socia, $3(2), 215-233$.

Quintanal, J., Melendro, M., Rodríguez, A. E. y Marí, S. (2016). Acción socioeducativa con colectivos vulnerables. Universidad Nacional de Educación a Distancia.

Pérez de Guzmán, V., Trujillo,J. F. y Bas, E. (2020). La educación social en España: Claves, definiciones y componentes contemporáneos. Revista Colombiana de Ciencias Sociales, 11(2), 632-658. https://doi.org/10.21501/22161201.3095

Ruiz-Corbella, M. y García-Gutiérrez, J. (2019). Aprendizaje-servicio. Los retos de la evaluación. Narcea.

Santos, M., Crespo, J., Lorenzo, M. y Godás, A. (2012). Escuelas e inmigración en España: ¿Es inevitable la segregación? Cultura y Educación, 24(2), 193-205. https://doi.org/10.1174/113564012804932128

Sapon, M. (2013). La inclusión real: Una perspectiva de justicia social. Revista de Investigación en Educación, 11(3), 71-85.

Shaeffer, S. (2019). Inclusive education: A prerequisite for equity and social justice. Asia Pacific Education Review, 20(2), 181-192. https://doi.org/10.1007/s12564-019-09598-w

Terrón, T., Cárdenas R. y Rodríguez, R. (2017). Educación intercultural inclusiva. Funciones de los/as educadores/as sociales en instituciones educativas. Pedagogía social. Revista Interuniversitaria, 29, 25-40. https://doi.org/10.7179/PSRI_2017.29.02

UNESCO. (1999). Los siete saberes necesarios para la educación del futuro. Santillana.

Vargas, Y. y García, V. M. (2021). El profesor tutor en los programas de tutoría entre iguales en la universidad. South Florida Journal of Development, 2, 2428-2450. https://doi.org/10.46932/sfjdv2n2-103

Vences, M. P. (2020). La equidad, elemento esencial para la inclusión en educación. UVserva, 10, 139-156.

Viana-Orta, M. I., Senent, J. M. y Camacho, G. (2019). La titulación de educación social en España. Análisis comparado de algunas de sus características. Educació Social. Revista d’Intervenció Socioeducativa, 73, 157-172.

Vila, E., Cortés, P. y Martín, V. M. (2020). Los educadores y educadoras sociales en los centros educativos de Andalucía: Perfil y desarrollo profesional. REICE. Revista Iberoamericana sobre Calidad, Eficacia y Cambio en Educación, 18(1), 47-64. https://doi.org/10.15366/reice2020.18.1.003

Vilar, J. (2018). Elementos de reflexión para el futuro próximo de la educación social. Revista d'Intervenció Socioeducativa, 70, 17-38.

Wehmeyer, M (2009). Autodeterminación y la tercera generación de prácticas de inclusión. Revista de Educación, $349,45-67$.

Zarzuela, A. y García, M. (2021). Aprendizaje-servicio crítico en la formación inicial del profesorado a través de un itinerario curricular. Holos, 37(2), 1-20. 
Zayas, B., Gozálvez., V. y Gracia, J. (2018). La dimensión ética y ciudadana del aprendizaje servicio: Una apuesta por su institucionalización en la educación superior. Revista Complutense de Educación, 30(1), 1-15.

https://doi.org/10.5209/RCED.55443

Zugaza, U. (2017). Claves hacia el empoderamiento de las mujeres gitanas: Un análisis desde el punto de vista de la interseccionalidad. Investigaciones Feministas: Papeles de Estudios de Mujeres, Feministasy de Género, 8(1), 203-222. https://doi.org/10.5209/INFE.54459

\section{Breve CV de los/as autores/as}

\section{Ana Amaro Agudo}

Actualmente Profesora Ayudante Doctora en la Facultad de Ciencias de la Educación (Universidad de Granada). Doctora en Pedagogía por la Universidad de Granada. Cuenta en su haber con la publicación de un importante número de artículos científicos en revistas nacionales e internacionales, así como varios libros, fundamentalmente relacionados con la inclusión y colectivos vulnerables. Ha participado como equipo investigador en múltiples proyectos de investigación $\mathrm{I}+\mathrm{D}$ de carácter nacional e internacional relacionados con los valores y la inclusión social, así como Coordinadora de varios proyectos de innovación y del Vicerrectorado de Igualdad, Inclusión y sostenibilidad de la Universidad de Granada. Email: anaamaro@ugr.es

ORCID ID: https://orcid.org/0000-0001-8033-0488

\section{Andrés Soriano Díaz}

Doctor en Filosofía y Ciencias de la Educación, es profesor titular en el Departamento de Pedagogía de la Facultad de Ciencias de la Educación de la Universidad de Granada y miembro del Instituto de la Paz y los Conflictos (IPAZ) de la misma Universidad. Pertenece al grupo de investigación "Valores emergentes, educación social y políticas educativas” (HUM-580). Ha participado como investigador y dirigido varios proyectos de carácter nacional e internacional. Su actividad investigadora se desarrolla en el ámbito de las violencias, los valores y en la educación para la paz. Cuenta en su haber con la publicación de múltiples artículos en revistas de impacto, así como varios libros en editoriales de prestigio, todo ello relacionado con las problemáticas educativas y sociales. Email: asoriano@ugr.es

ORCID ID: https://orcid.org/0000-0002-7782-1814

\section{Nazaret Martínez-Heredia}

Doctora en Ciencias de la Educación por la Universidad de Granada. Actualmente Profesora del Departamento de Pedagogía en la Facultad de Ciencias de la Educación (Universidad de Granada, España). Anteriormente FPU (Formación de Profesorado Universitario) y Contratada Posdoctoral en el mismo Departamento. Pertenece al grupo de investigación "Valores emergentes, educación social y políticas educativas” (HUM-580) y es miembro del Instituto de la Paz y los Conflictos (IPAZ) de la Universidad de Granada. Ha publicado un gran número de artículos científicos en revistas nacionales e internacionales de alto impacto y ha participado en numerosos proyectos locales, nacionales e internacionales. Desde 2016 su principal actividad se ha centrado en valores, pedagogía social y personas mayores. Email: nazareth@ugr.es

ORCID ID: https://orcid.org/0000-0001-7029-7127

\section{Gracia González-Gijón}

Doctora en Pedagogía por la Universidad de Granada. Profesora del Departamento de Pedagogía de la Facultad de Ciencias de la Educación, Universidad de Granada, España. Secretaria del Departamento de Pedagogía desde 2021. Pertenece al grupo de investigación "Valores emergentes, educación social y polí- 
ticas educativas" (HUM-580) y es miembro del Instituto de la Paz y los Conflictos (IPAZ) de la Universidad de Granada. Participa en la Red iberoamericana de Cooperación para la Democratización Familiar y Comunitaria (REDIFAC). Sus líneas de investigación se centran en valores, violencia en las relaciones de pareja jóvenes, democratización familiar y diversidad cultural. Actualmente es investigadora principal de varios proyectos nacionales e internacionales cuyos resultados se han divulgado revistas científicas de reconocido prestigio. Email: graciag@ugr.es

ORCID ID: https://orcid.org/0000-0001-9246-167X 